\title{
Dendritic supports in organic synthesis
}

\author{
Robertus J.M. Klein Gebbink**, Cornelis A. Kruithof ${ }^{1}$, \\ Gerard P.M. van Klink, Gerard Van Koten* \\ Debye Institute, Department of Metal-Mediated Synthesis, Utrecht University, Padualaan 8, 3584 CH Utrecht, \\ The Netherlands
}

\begin{abstract}
An overview is presented of the recent developments in the use of dendritic supports in organic synthesis. Examples are presented of the application of dendritic supports in both liquid- and solid-phase organic synthesis. In liquid-phase synthesis, soluble dendrimers are used as the substrate support, while in solid-phase synthesis, 'dendronized' insoluble resins are used for this purpose. Selected examples of the synthesis of compound libraries on dendritic supports via combinatorial techniques are discussed. (c) 2002 Elsevier Science B.V. All rights reserved.
\end{abstract}

Keywords: Dendrimers; Organic synthesis; Supports

\section{Introduction}

The use of insoluble resins as supports for substrates in organic synthesis has been increasingly explored since the pioneering work of Merrifield $(1963,1969)$. The development of this strategy of solid-phase organic synthesis (SPOS) was initially spurred by the facilitated product purification; the reaction products remain bound to an

\footnotetext{
* Corresponding author. Tel.: +31-30-253-3120; fax: +3130-252-3615.

E-mail address: g.vankoten@chem.uu.nl (G. Van Koten).

** Corresponding author. Tel.: +31-30-253-3120; fax: +3130-252-3615.

E-mail address: r.j.m.kleingebbink@chem.uu.nl (R.J.M. Klein Gebbink).

${ }^{1}$ Based on an internal report submitted in partial fulfilment of the requirements for a MSc degree.
}

insoluble resin and reagent excesses are simply removed by repeated rinsing. The SPOS process is presented in Fig. 1. An insoluble resin (most often cross-linked polystyrene (PS)) is functionalized with a linker moiety carrying a functional group to which the substrate is connected. A reaction or sequence of reactions is carried out to convert the supported substrate into the supported product and excess reagents are washed away after each step. Finally, selective cleavage yields pure product in solution and the insoluble support, which is recycled for further use. The original method of Merrifield's dipeptide synthesis on a polymer support has since been extended to basically every type of reaction in organic chemistry, including polypeptide, -saccharide, and -nucleotide synthesis. Because supported synthesis is very amenable to automation, it has become 


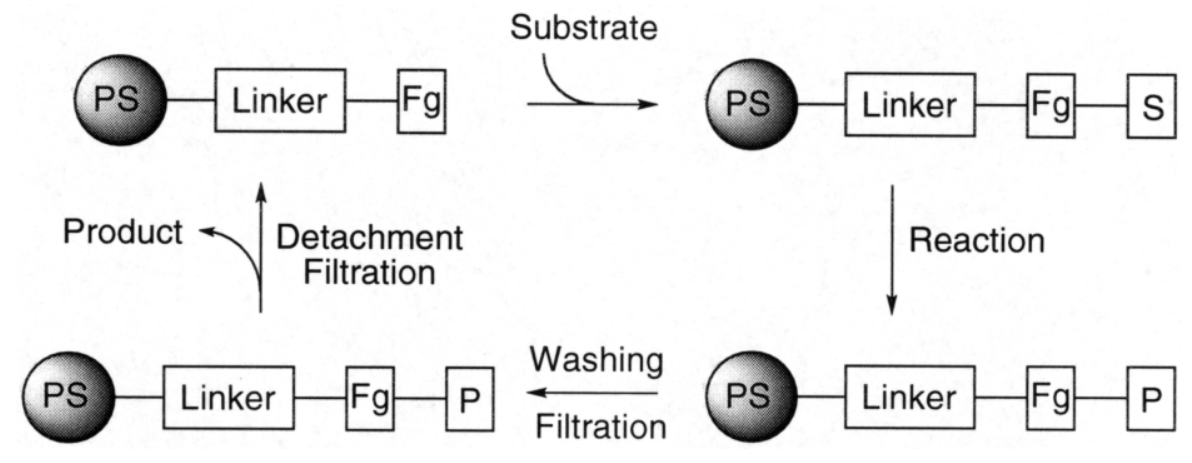

Fig. 1. SPOS principle.

of great importance, leading e.g. to the development of combinatorial chemistry, often applied in pharmaceutical and agrochemical research (for selected reviews see Thompson and Ellman, 1996; Pirrung, 1997; Franzén, 2000). In pharmaceutical chemistry, combinatorial chemistry offers the possibility of drug discovery at high speed using mix-and-split or one-pot synthesis methods combined with high-throughput screening of a large number of compounds.

The advantages of solid-supported synthesis are multiple: (i) reactants can be used in excess to drive reactions to completion and hence (ii) high yields can be obtained; (iii) the loaded support can easily be separated from the reactants, which considerably simplifies and speeds up reaction procedures; and overall (iv) the synthetic procedures allow automation. However, solid-phase organic synthesis also has its restrictions. Due to the bi-phasic nature of the process (solution phase/solid phase), reactions can proceed relatively slow in comparison to the corresponding solution-phase reactions. In addition, the heterogeneous nature can lead to non-linear reaction kinetics. In order to develop efficient chemistry on supported substrates, standard organic liquidphase reaction conditions have to be translated to solid-phase chemistry. The accessibility of reagents and the compatibility of the support with the reaction conditions applied are major issues in the field of solid-supported synthesis. A major disadvantage in most SPOS strategies is the difficulty of monitoring the progress of single reaction steps. In most cases, standard spectroscopic analysis of supported intermediates is at least tedious, if not impossible. The additional steps needed to bind and release the product from the support present another general drawback of supported synthesis. The development of suitable linkers and attach/detach procedures are timeconsuming tasks.

To overcome the disadvantages of SPOS, or at least some of them, several alternative processes have been developed in recent years (Ley et al., 2000 and references therein). A closely related process makes use of supported reagents, including catalysts. This process represents an inverse approach to SPOS; instead of supporting the organic substrate, the reagents required in the organic transformation of the substrate are now supported. Although this approach eliminates the binding and release of substrates from supports, it still involves bi-phasic chemistry. A major advantage of this approach is that it allows standard spectroscopic monitoring of ongoing chemistry. Similarly, supported scavengers or sequesters have been developed, which remove reagent excesses from solution reaction mixtures, thereby 'cleaning up' an organic reaction. The use of both supported reagents and scavengers has recently been most conveniently reviewed by Ley et al. (2000).

An intriguing alternative for the use of insoluble supports in SPOS is the use of soluble supports in so-called liquid-phase organic synthesis (LPOS). This approach avoids the problem of solid-liquid phase translations and gives all the benefits of homogeneous reactions (Gravert and Janda, 1997; Toy and Janda, 2000). Due to the 
more facile accessibility of the substrates in solution, usually no translation to solid-phase chemistry of earlier developed reaction methods is required, and therefore standard reaction conditions can be applied. Another attractive aspect is that, in contrast to solid-phase synthesis, supported intermediates can often be analyzed by standard spectroscopic means. The course of the reaction can be closely followed and re-directed if necessary. A crucial step in the success of the LPOS approach is the development of a simple and effective separation procedure. Some of the purification methods developed are solvent- or heat-induced precipitation, followed by filtration/decanting, membrane filtration and size-exclusion chromatography (SEC). The soluble polymers most often used in LPOS are poly(ethylene glycol) (PEG), (linear) polystyrene (PS) and poly(vinyl alcohol). One of the major considerations in LPOS is the compromise that has to be made between the loading of the support and its overall solubility properties. High loading capacity can lead to non-equivalent reactivity of the supported substrates, while solubility properties can become more substrate- than support-like, leading to separation problems. On the other hand, large amounts of support material have to be used when the loading capacity is low. The LPOS approach has been extended to soluble supported reagents, scavengers and catalysts (Bergbreiter, 1998; Wentworth and Janda, 1999).

Dendrimers are a class of perfectly branched, tree-like polymers concentrically built up around a central core unit (Tomalia, 1993; Fréchet, 1994; Newkome et al., 1996; Vögtle, 1998, 2000). In contrast to regular polymers, these globular cascade molecules possess a highly ordered and well-defined structure, which provides them with special chemical and physical properties (Bosman et al., 1999). Dendrimers can be prepared via two types of iterative syntheses. In the divergent synthesis, branching units are connected to a core molecule in a stepwise manner, such that the dendrimer is extended layer by layer at its periphery. The dendritic polymer is built from the inside out to yield different generations of the dendrimer along the synthetic pathway. In the convergent synthesis, individual dendritic wedges or dendrons with a single reactive group at its socalled focal point are constructed. The dendrimer is constructed in the last step of the synthesis by the combination of several wedges with an oligovalent core unit. In this manner, dendrimers are constructed from the outside in. Research in dendritic species has substantially grown in the past decades. Their applications cover many aspects of chemistry, for example catalysis, self-assembly, host-guest chemistry, sensory materials and drug delivery systems.

The number of functional groups on the periphery of a dendrimer is multiplied from generation to generation, leading to functional group amplification as the dendrimer increases in size. The large spherical shape of dendrimers, additionally, allows simple purification methods, such as SEC and ultra-filtration. These two aspects could make dendrimers very useful supports in organic synthesis. A further advantage of soluble dendritic supports is the possibility of investigating supported reaction intermediates with standard analytical methods, such as NMR, IR and MS, due to the highly ordered and repetitive build-up of dendrimers.

This report describes representative examples of the use of (i) dendritic molecules and building blocks as supports in LPOS and (ii) dendritic wedges attached to insoluble resins as high-loading solid supports in SPOS. Both the synthesis of the dendritic supports themselves and the synthesis of selected organic compounds (and libraries thereof) on the supports are presented in some detail. The use of dendritic catalysts has recently been reviewed and is not included in this report (Kreiter et al., 2001; Oosterom et al., 2001; Kleij et al., in press).

\section{Dendrimers as liquid-phase supports}

The use of dendrimers as substrate supports for organic synthesis was first reported by Kim et al. (1996) at Merck Research Laboratories. In this initial literature report, the authors recognized the full advantages that this approach could offer compared to 'conventional' solid support synthesis (vide supra). Not only was both the synthesis 
of an organic substrate on a dendritic support and its release from the support described, but the first synthesis of a small library of substrates using a dendritic support was also demonstrated. The authors coined the term dendrimer-supported combinatorial chemistry (DCC) for this process. The support that was used in these studies consisted of a starburst poly(amidoamine) dendrimer (PAMAM) of generation 1.0, to which 4-hydroxymethylbenzoic acid (HMBA) linkers were connected (Fig. 2). A small series of indole derivatives was synthesized on this support via the Fischer indole synthesis in excellent yields and purity. SEC on Sephadex LH-20 could purify all intermediates within $15 \mathrm{~min}$ and without special pre-treatment of the column. It was claimed that these columns could be used over a dozen times. Most interestingly, all dendritic intermediates could be analyzed using both conventional ${ }^{1} \mathrm{H}$ and ${ }^{13} \mathrm{C}$ NMR spectroscopy and mass spectrometry. Applying mix-and-split techniques, a small library of 27 indole derivatives was generated using this concept. Following this proof of principle, several other companies filed patents for the use of dendritic supports in organic synthesis, and more specifically, combinatorial and highthroughput chemistry.

By making use of carbosilane dendrimers, Van Koten and co-workers showed the use of dendritic supports in syntheses involving reactive organometallic intermediates (Hovestad, 1999; Hovestad et al., 1999). Carbosilane dendrimers

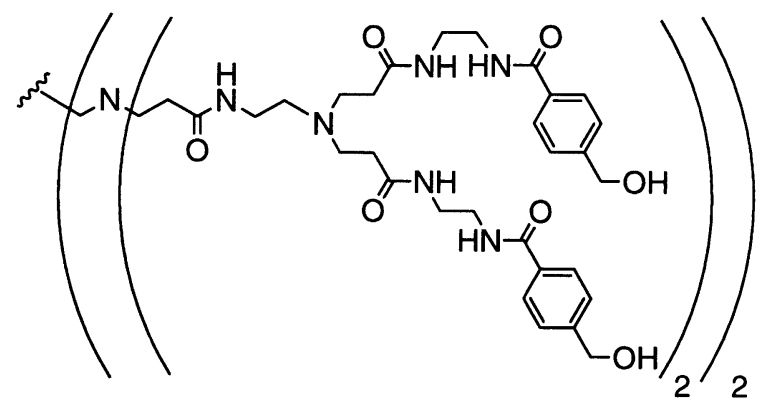

Fig. 2. PAMAM-derived dendritic support used by Kim.

have the striking advantage of being inert to most reagents used in organic synthesis. Another advantage of their approach is that no cleavage reaction of the product was required because the dendritic species was used as a leaving group. The chiral dendritic support used in these studies was obtained from the corresponding chloro-silane dendrimers via organolithium chemistry (Fig. 3). The substrate was then loaded on the support via a simple esterification reaction. Lithiation using lithium diisopropylamide (LDA), followed by the addition of $\mathrm{ZnCl}_{2}$, gives the zinc enolate, which reacts in situ with an imine to form the free $\beta$-lactam and the trimethyl silane (TMS)-protected dendritic support. Product formation was highly trans-selective, with diastereomeric excess (de) values up to $95 \%$, although enantiomeric excess (ee) values were modest $(\sim 30 \%)$. Besides recycling of the support via SEC, preliminary retention experiments also showed that the den-
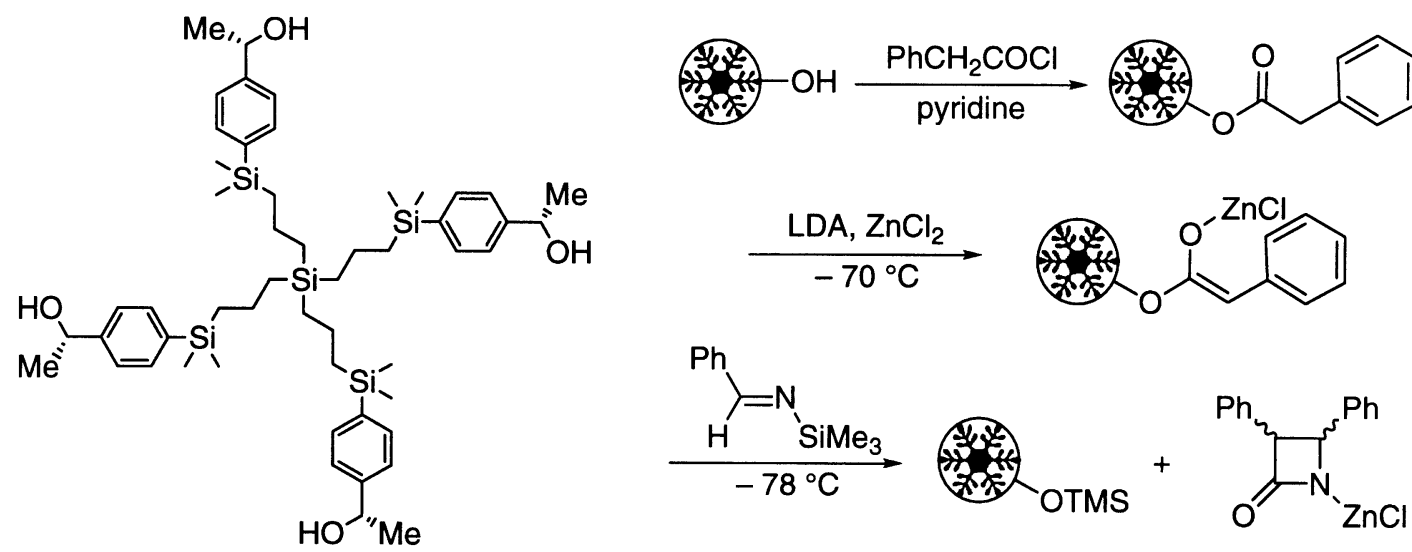

Fig. 3. Synthesis of $\beta$-lactams on a carbosilane dendrimer support developed by Van Koten. 
dritic support is of sufficient size to be retained to a large extent by a nanofiltration membrane (MPF-60 NF). This approach was later extended to Suzuki cross-coupling reactions, in which the halide derivative of the aryl substrate from the above example was used (Hovestad et al., 2000a). This example illustrates the accessibility of multi-step synthesis of dendrimer-supported compounds.

The use of dendritic supports in materials synthesis was demonstrated by Fréchet and coworkers (Malenfant et al., 1999). Aliphatic ether dendrons were used not only as support, but also as a solubilizing agent in the synthesis of oligoand polythiophenes with minimal substitution (Malenfant and Fréchet, 2000). These compounds are interesting because of their electrical conductivity properties. Due to the poor solubility of oligo- and polythiophenes, solubilizing substituents are often attached to these thiophenes. However, substitution of the thiophene backbone results in larger bandgaps of the polythiophene material. Second- and third-generation aliphatic ether dendrons were attached to either a $2,2^{\prime}$-bithiophene or to a 2,5-dibromo monothiophene (Fig. 4). Repeated $N$-bromo-succinamide (NBS) bromination and Stille coupling reactions led to either mono- or bi-directional extension of the supported (oligo)thiophenes. No cleavage of the oligo- or polythiophenes from the dendritic support was reported.

Van Koten and co-workers have also used car- bosilane dendrimers as soluble supports for the synthesis of poly(methyl methacrylate) (pMMA) (Hovestad et al., 2000b). Dendrimers of the type shown in Fig. 3 were functionalized with 2bromo-isobutyryl groups and used as oligo-valent initiators in the copper(I) bromide $/ N$-( $n$-octyl)2-pyridylmethanimine-mediated living-radical polymerization of methyl methacrylate. The overall rate of polymerization was slower than in the case of a mono-valent initiator, which was ascribed to intermolecular termination reactions. The star polymers obtained via this procedure have molecular weights of up to 33000 with polydispersity values below 1.3. The individual pMMA chains were not detached from the dendritic support. Whereas the star-shaped polymeric products are interesting in terms of their material properties, this approach also illustrates the possible use of soluble dendritic supports in polymer synthesis.

Several types of soluble polymers have been used as substrate supports in organic synthesis (LPOS). The main advantage of these soluble polymers in contrast to insoluble supports is that all the chemistry takes place in one phase, and thus general solution procedures can be applied without alterations. A major drawback of these supports lies in the low substrate loading that can be achieved. One way of increasing the substrate loading of soluble polymers is to incorporate one or more dendritic segments. This approach has lead to end-group 'dendronized' polymers on the

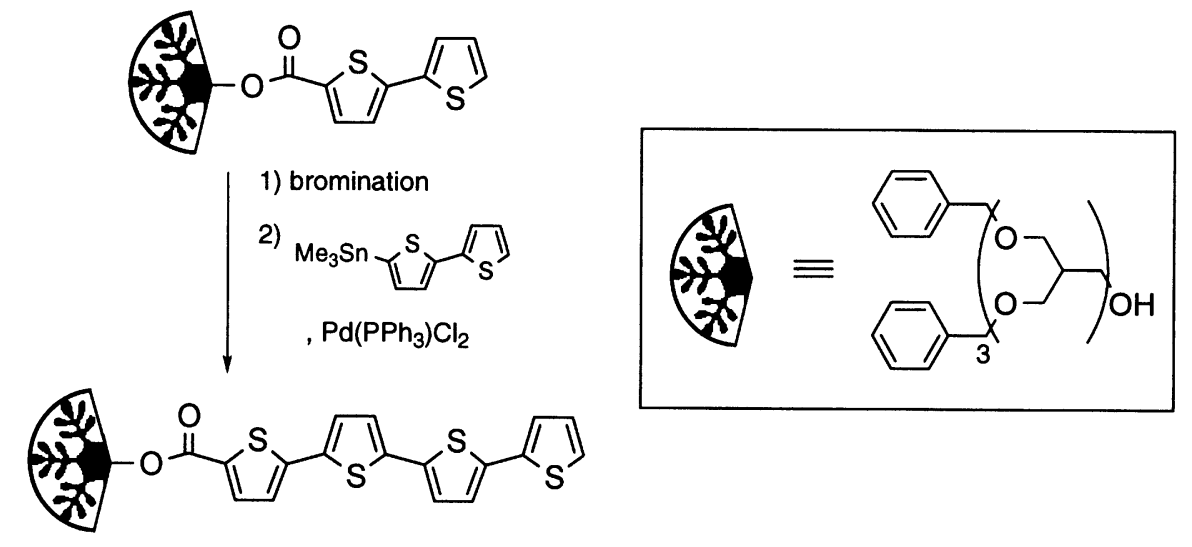

Fig. 4. Dendron-supported synthesis of oligothiophenes by Fréchet. 


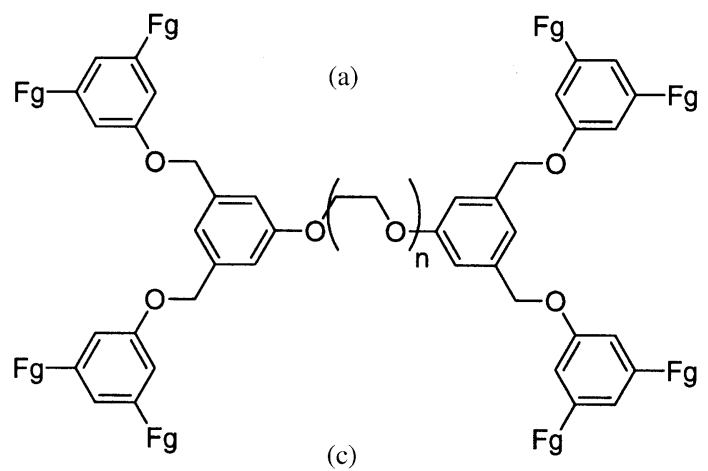

(c)

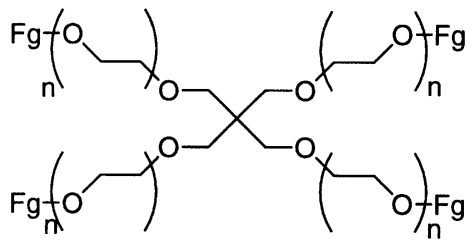

(b)

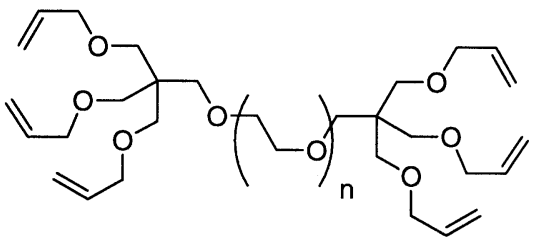

(d)

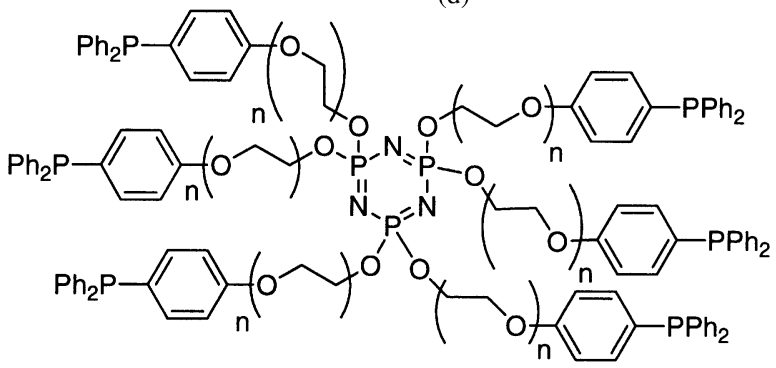

Fig. 5. Poly(ethylene glycol)-based, soluble supports (Fg, functional group).

one hand, and to single-branched, oligo-valent polymers on the other hand (Fig. 5).

An early example of this approach was reported by Cozzi and co-workers (Benaglia et al., 1998). Via the attachment of isophthalate groups to both ends of $\mathrm{PEG}_{4600}$ tails, a doubling of the reactive groups on the soluble polymer was achieved. The isophthalate units allow entry into several types of functional groups via standard organic reactions. Use of a tetra-imine support led to the synthesis of supported azetidinones via the Staudinger reaction. Cleavage and isolation of the reaction products from the supports only proceeded with moderate yield. A further doubling of the number of reactive groups was achieved after treatment of the tetra-benzyl chloride support with isophthalate, leading to a PEG polymer bearing poly(benzyl ether) dendrons, socalled 'inverted' Fréchet-wedges (Fig. 5a). The loading of functional groups in these PEG-based supports increased from 0.43 to 0.86 to $1.73 \mathrm{mmol}$ $\mathrm{g}^{-1}$, for dihydroxy-, generation 1-, and generation 2-PEG ${ }_{4600}$, respectively (Wentworth and Janda, 1999).

A PEG polymer, end-capped with allylated bis-pentaerythritol functionality, was used as the soluble support in the synthesis of di- and oligosaccharides by Narvor and co-workers (Lubineau et al., 2000; Fig. 5b). In these studies, the thiosugar substrate was attached to the support by radical coupling of the thiol group onto the double bond. According to MALDI-TOF analysis, full substrate loading was not obtained with this procedure. An enzymatic galactosylation to form a disaccharide was carried out on the support using the enzymes galactosyl transferase and $\mathrm{UDP}$-glucose/UDP-glucose 4-epimerase, the progress of which was monitored by means of NMR analysis of the soluble supported material. The supported disaccharide was then fucosylated using fucosyltransferase III (Fuc TIII), followed by product detachment via treatment with mercury(II) trifluoracetate. Although the yields isolated were moderate, this example represents an interesting combination of the use of dendronized soluble polymers and biocatalysis in organic synthesis. The system was also tested for chemical manipulations; sulfatation of supported lactose was found to occur with approximately 60-70\% conversion, affording 3 '-sulfated lactose.

The Kim group has illustrated the use of oligovalent soluble supports in the synthesis of a li- 
brary of di- and trisubstituted guanidines (Chang et al., 1999). A series of tetra-PEG-appended pentaerythritols with PEG tails of varying length were used as supports in this study (Fig. 5c). To immobilize substrates, it was functionalized with an acid-labile Rink linker. Two $N$-(9-fluorenylmethoxycarboxyl) (Fmoc) amino acids, four isothiocyanates and four amine derivatives were used as starting materials in the generation of a 48-compound library (including 16 compounds without the Fmoc acid). Significant polymerization of the support was observed after cleavage of the product, presumably caused by cross-linking of the Rink linker. Interestingly, a semi-automated SEC method was used, for which a support recovery of approximately $95 \%$ was reported. Four parallel columns were used, which were connected to a syringe pump delivering the eluent. Purification was established within minutes, although the purity varied from 0 to $95 \%$. The loading capacity of these supports is two-fold greater than that of a single PEG chain, whereas the loading per gram of material is identical. This process was termed COSMOS (combinatorial synthesis on multivalent oligomeric supports) by the authors.

The use of supported reagents allows easy and fast removal of (costly) reagents from homogeneous reaction media. So far, insoluble reagents have been used in most cases. Janda and coworkers have pioneered the use of soluble supported reagents. Recently, they reported on an oligo-valent reagent derived from a PEGappended hexa-functional cyclotriphosphazene core (Reed and Janda, 2000; Fig. 5d). One of the advantages of this type of molecule is the nearly identical ${ }^{1} \mathrm{H}$ and ${ }^{13} \mathrm{C}$ NMR spectra to those of PEG, which allows NMR spectroscopic analysis of the bound intermediates. Therefore, the authors refer to this molecule as a 'Stealth Star'. The star-shaped support was functionalized with triphenylphosphine and tested in the Mitsunobu etherification of phenol with several alcohols. The yields were comparable to etherification reactions with mono-valent triphenylphosphine $\left(\mathrm{PPh}_{3}\right)$ functionalized PEG as support. Recycling of the resulting phosphine oxide support was possible by precipitation from diethyl ether (85\%). Regenera- tion of the $\mathrm{PPh}_{3}$ functionality was accomplished by reduction with alane without degradation of the cyclotriphosphazene core, which enabled recycling and reuse of the supported reagent.

\section{Dendronized solid-phase supports}

Traditionally, the supported synthesis of organic compounds takes place on insoluble polymer beads. Whereas this approach is successfully applied in a wide range of applications, a single bead often yields insufficient product for standard analysis methods, such as NMR and IR spectroscopy. The number of reactive groups on a bead can be increased via the attachment of dendritic fragments (dendrons) that bear similar reactive groups at their periphery. In this strategy, the dendron can be regarded as a linker, as well as a reactive group scaffold, which in addition gives the supported molecules more soluble-like properties due to its large size. Although this approach does not substantially increase the number of reactive groups per gram of support material, it does lead to a considerably higher loading per single bead.

Although resin-bound lysine dendrimers had been reported for multiple-antigenic peptide synthesis (Tam, 1988), this approach was basically developed by Bradley and co-workers. In one of their first reports on this issue, PAMAM dendrons were synthesized onto an insoluble polystyrene-poly(ethylene glycol) resin (e.g. TentaGel) (Swali et al., 1997). Build-up of the dendritic linkers was performed on the resin beads, i.e. generation after generation (divergent approach), instead of connecting pre-synthesized dendrons to the beads (convergent approach). In this manner, supported dendrons up to generation 4.0 were synthesized (Fig. 6a). The loading capacity of the highest generation material is 2.8 mmol $\mathrm{g}^{-1}$, which would amount to a single bead loading of approximately $9.6 \mathrm{nmol} \mathrm{bead}{ }^{-1}$ ! A typical feature of the loading aspect in this approach is the fact that from generation 3.0 to generation 4.0, the loading per gram of support only increases from 2.3 to $2.8 \mathrm{mmol}^{-1}$, whereas the loading per single bead doubles during this 
process. Upon treatment with $50 \%$ trifluoroacetic acid (TFA) the PAMAM dendrons could be cleaved from the support, albeit with a certain amount of additional PEG cleavage. The primary amine groups at the dendron periphery were functionalized with a super acid-sensitive linker 4-(4'-hydroxymethyl-3'-methoxy)phenoxybutyric acid (HMPB) and loaded with protected glycine (Gly) to synthesize the peptide Fmoc-LysGly-OH at the periphery of the dendrimer. Treatment with $1 \%$ TFA in $\mathrm{CH}_{2} \mathrm{Cl}_{2}$ led to the selective removal of the di-peptide from the dendronized support. The full advantage of this high-loading approach was shown in the synthesis of a library of tri- and hexa-peptides using splitand-mix library applications on related PS-PEG-PAMAM supports of generation 2.0 (Wells et al., 1998, 1999). Analysis of the peptide products could be achieved using conventional ${ }^{1} \mathrm{H}$ NMR, HPLC and ES-MS techniques on product obtained from a single bead. Similar chemistries on these so-called DendroGels were later described for Mitsunobu coupling reactions to yield amino acid-derived aryl ethers (Basso et al., 2000a) and for the synthesis of a small library of amidines of pharmaceutical interest (Basso et al., 2000b). As an alternative to the PAMAM dendrons, the use of 'inverted' Fréchet-type dendrons was also explored (Basso et al., 2001). Starting from either a glycine-Rink-modified polystyrene resin or from a hydroxymethylpolystyrene resin, several generations of poly(benzyl ether) dendrons were synthe- sized (Fig. 6b). Generation 3-dendronized resins were further modified with 4-hydroxymethyl aryl ethers, which led to a Wang-type dendronized resin with eight Wang linkers per dendritic wedge. The hexa-peptide Leu-enkephaline-Lys was synthesized on this resin, with $66 \%$ yield isolated after release from the support.

In a separate paper, Fromont and Bradley (2000) reported on the development of a dendronized support with substantially higher loading capacity then the previous examples. Several generations of PAMAM-like dendrons were synthesized on aminomethylpolystyrene resins of different bead sizes, leading to resins with loading capacity from 36 to $200 \mathrm{nmol} \mathrm{bead}^{-1}$ ! The large increase in functional group loading was achieved by means of a tri-functional branching unit in the dendritic structure, compared to a bi-functional branching unit in the PAMAM and Fréchet-type structures (Fig. 6c). The unique properties of these high-loading resins are exemplified by their swelling tendency in solution (up to $9 \mathrm{ml} \mathrm{g}^{-1}$ ). The swelling of these materials is much more pronounced than for the DendroGel materials. Use of the high-loading resins in supported synthesis was presented in the synthesis of biphenyls via the Suzuki coupling and of tri-peptides. Notably, the amount of product material obtained from a single bead is more than sufficient for routine analysis (e.g. NMR).

Chan and co-workers have developed an alternative, yet related system using a tri-amino ester

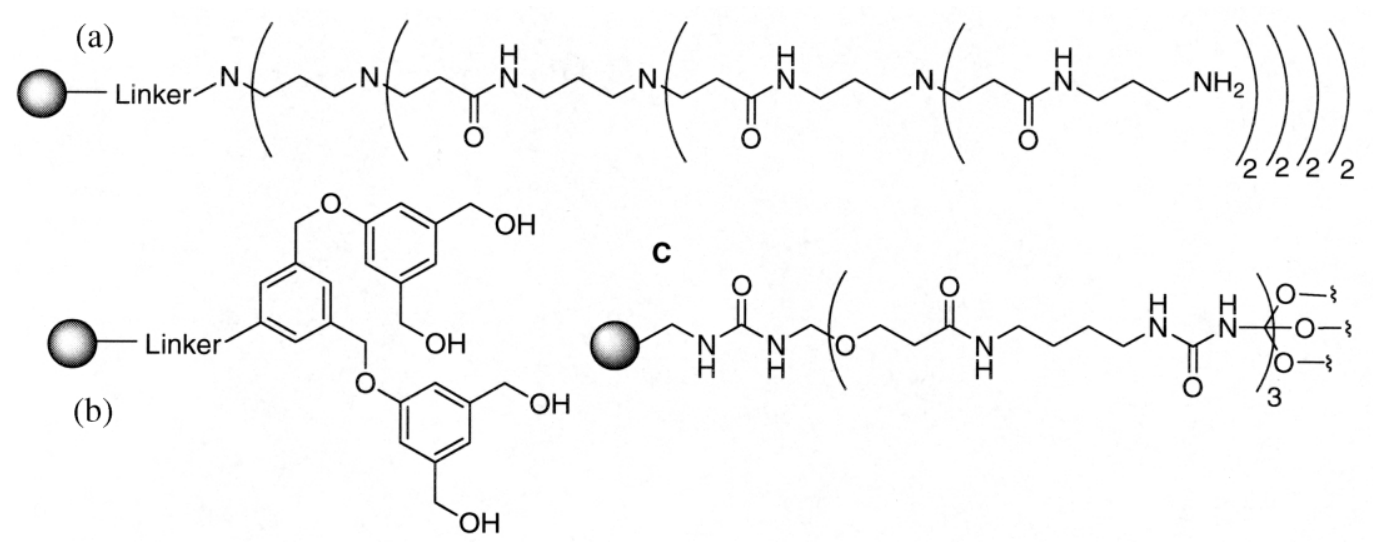

Fig. 6. Dendronized solid supports developed by Bradley. 
building block for the construction of dendronized PS-beads (Mahajan et al., 1999). By means of a protection/deprotection repeated synthesis, two generations of triple-branched dendrons were attached to aminomethylpolystyrene (Fig. 7a). These resins seem comparable to the triple-branched PAMAM resins in terms of loading capacity and swelling (solution) behavior, but can have the advantage of reactive group differentiation. Using a Rink amide linker, 2-naphthalenesulphonamide $(84 \%)$ and $N$-phenylalaninamide $(87 \%)$ were successfully synthesized on these resins. Product release by treatment with TFA in water proceeded without damaging the dendritic resin.

Along with the role of resins as substrate supports in organic synthesis, insoluble resins also find wide use as scavenger supports. In this case, synthesis is carried out in solution and reaction by-products and/or reagent excesses are selectively scavenged out of solution by an insoluble resin. Marsh et al. (2001) have developed a highloading dendronized resin for use as a nucleophile or an acid scavenger (Fig. 7b). The triazine dendritic wedges were grown on a Wang resin and could be cleaved from the resin (5\% TFA in $\mathrm{CH}_{2} \mathrm{Cl}_{2}$ ) for analysis by NMR, IR and MS techniques. Two examples of the use of these scavengers have been presented in the acylation and the tosylation of benzylic amines. The advantage of this type of resin over its polymeric counterparts is the reduced amount of supported scavenger required to clean up a typical product-containing solution. This is particularly favored when used in the synthesis of small amounts of compounds, a situation often encountered in combinatorial synthesis. The commercially available PS-Tris resins can be thought of as the prototype for these specialty scavengers. Tris resins were earlier used as, e.g. (thio)isocyanate quenchers in (thio)urea synthesis (Booth and Hodges, 1997), combined imine/diene scavengers in the synthesis of dihydropyridone libraries, and as acid chloride scavengers in acylation of aminopiperidines (Creswell et al., 1998).

\section{Outlook}

The main advantage of dendrimers as unimolecular supports is the combination of solution chemistry with relatively high loading capacity. Although loading capacity per gram of material might not be that high, the loading capacity per supporting molecule could be 10 s of moles for higher dendrimer generations! However, one of the general features of these higher generation dendrimers is that they show considerable steric congestion of peripheral groups. This could lead to non-equivalent reactivity of the supported substrates, e.g. due to reagent accessibility problems. Therefore, similar considerations have to be made as in LPOS on linear polymers and in SPOS. In the case of a dendritic support, there will be an optimal dendrimer generation that combines both a high loading capacity per single molecule and good reactivity profiles for the supported substrates. Similar considerations apply for den-

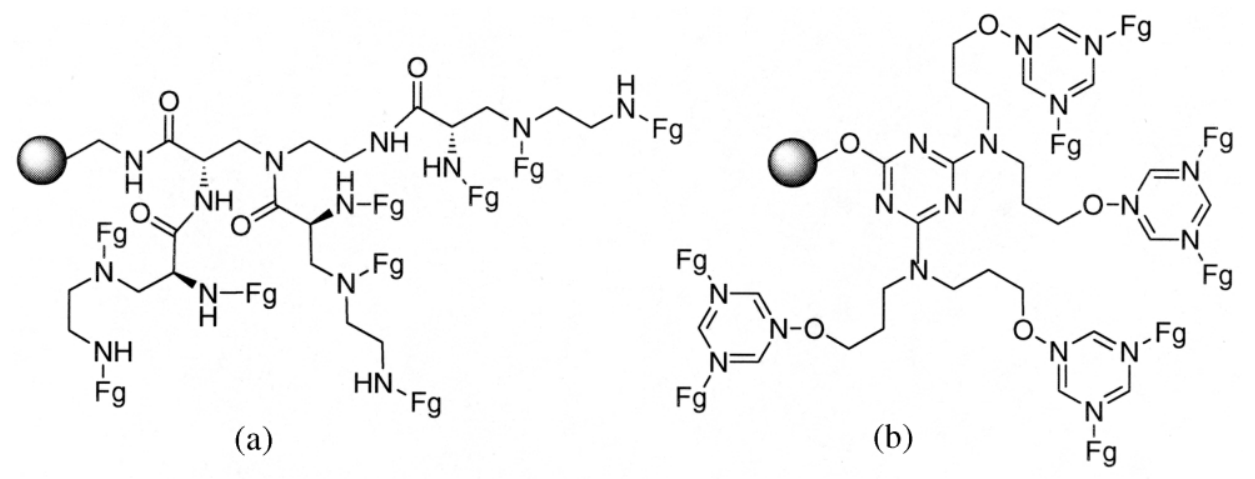

Fig. 7. Dendronized solid supports developed by (a) Chan and (b) Marsh (Fg, functional group). 
dronized resins. Also in this case, higher dendron generations could provide an increased loading capacity per bead, and even a more solution-like reactivity of the supported substrates. Again, peripheral congestion could lead to incomplete reaction profiles and unwanted side reactions.

An alternative for the dendrimer supports, with ideally perfect molecular architectures, are represented by supports derived from hyper-branched polymers. These polymers can most often be prepared in a single step and in large quantities, compared to the often tedious and lengthy dendrimer synthesis. Due to the highly branched structure, hyper-branched polymers contain a large number of terminal groups on the surface. The properties of hyper-branched polymers have recently been reviewed, as well as their initial application as supports in organic synthesis (Haag, 2001). A selected example is represented by the work of Kantchev and Parquette (1999). A hyper-branched polyester, the so-called Boltron polymer (Fig. 8), was used for the synthesis of a di-saccharide. A $40 \%$ loading of the polymer was used to avoid steric crowding at the periphery. Purification of the supported product and of the support after product release was carried out via SEC, although precipitation was considered more convenient. In the development of hyperbranched supports, similar aspects have to be faced as for other supports, i.e. loading/reactivity considerations, means of support recovery, and the extent to which reactions proceed and supports can be isolated.

So far, the different types of dendrimers and dendrons that have been used in supports for organic synthesis are rather limited. Aside from the popular PAMAM dendrimers and Fréchet

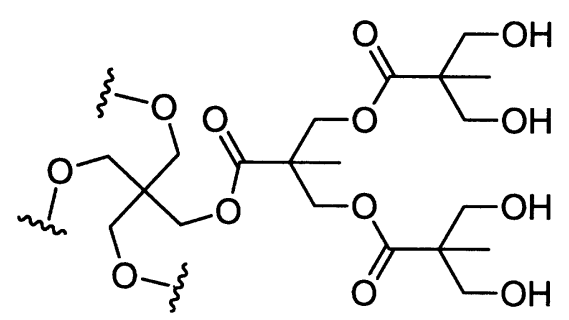

Fig. 8. Idealized representation of the hyperbranched Boltron polymer. wedges, not many other types of dendritic materials (e.g. carbosilane dendrimers) have been explored. Research in the field of LPOS has shown that the nature of a support determines to a large extent its applicability in solution-phase syntheses. Not only does it determine the types of chemistry that can be carried out (e.g. PEG does not allow organometallic and anion chemistry), but it also determines the ease of characterization of supported intermediates, and more importantly, the success of isolation of the product and recovery of the support. The further development of dendritic supports that perform much better on these aspects will therefore represent an important and exciting future endeavor.

\section{Acknowledgements}

The authors would like to thank all their coworkers and students who have contributed to the work described in this paper. Utrecht University, CW-NWO, STW-NWO and the National Research School Combination Catalysis (NRSC-C) are acknowledged for continuous financial support.

\section{References}

Basso, A., Evans, B., Pegg, N., Bradley, M., 2000a. Solid-phase synthesis of aryl ethers on high loading dendrimer resin. Tetrahedron Lett. 41, 3763-3767.

Basso, A., Pegg, N., Evans, B., Bradley, M., 2000. Solid-phase synthesis of amidine-based GP IIb-IIIa antagonists on dendrimer resin beads. Eur. J. Org. Chem. 3887-3891.

Basso, A., Evans, B., Pegg, N., Bradley, M., 2001. Solid-phase synthesis of aryl-ether dendrimers. Chem. Commun. 697-698.

Benaglia, M., Annunziata, R., Cinquini, M., Cozzi, F., Ressel, S., 1998. Synthesis of new poly(ethyleneglycols) with a high loading capacity. J. Org. Chem. 63, 8628-8629.

Bergbreiter, D.E., 1998. The use of soluble polymers to effect homogeneous catalyst separation and reuse. Catal. Today 42, 389-397.

Booth, R.J., Hodges, J.C., 1997. Polymer-supported quenching reagents for parallel purification. J. Am. Chem. Soc. 119, 4882-4886.

Bosman, A.W., Janssen, H.M., Meijer, E.W., 1999. About dendrimers: structure, physical properties and applications. Chem. Rev. 99, 1665-1688.

Chang, J., Oyelaran, O., Esser, C.K. et al., 1999. Synthesis of 
di- and trisubstituted guanidines on multivalent soluble supports. Tetrahedron Lett. 40, 4477-4480.

Creswell, M.W., Bolton, G.L., Hodges, J.C., Meppen, M., 1998. Combinatorial synthesis of dihydropyridone libraries and their derivatives. Tetrahedron 54, 3983-3998.

Franzén, R.G., 2000. Recent advances in the preparation of heterocycles on solid supports: a review of the literature. J. Comb. Chem. 2, 195-214.

Fréchet, J.M.J., 1994. Functional polymers and dendrimers: reactivity, molecular architecture and interfacial energy. Science 263, 1710-1715.

Fromont, C., Bradley, M., 2000. High-loading resin beads for solid-phase synthesis using triple-branching symmetrical dendrimers. Chem. Commun. 283-284.

Gravert, D.J., Janda, K.D., 1997. Organic synthesis on soluble polymer support: liquid-phase methodologies. Chem. Rev. 97, 489-509.

Haag, R., 2001. Dendrimers and hyperbranched polymers as high-loading supports for organic synthesis. Chem. Eur. J. 7, 327-335.

Hovestad, N.J., 1999. Periphery Functionalized Dendrimers: Organic Synthesis and Homogeneous Catalysis. Utrecht University. PhD thesis.

Hovestad, N.J., Jastrzebski, J.T.B.H., Van Koten, G., 1999. Carbosilane dendritic species as soluble supports in synthesis. An example: the metal-mediated synthesis of $\beta$-lactams. Polym. Mater. Sci. Eng. 80, 53-54.

Hovestad, N.J., Ford, A., Jastrzebski, J.T.B.H., Van Koten, G., 2000a. Functionalized carbosilane dendritic species as soluble supports in organic synthesis. J. Org. Chem. 65, 6338-6344.

Hovestad, N.J., Van Koten, G., Bon, S.A.F., Haddleton, D.M., 2000 b. Copper(I) bromide $/ N$-( $n$-octyl)-2-pyridylmethanimine-mediated living-radical polymerization of methyl methacrylate using carbosilane dendritic initiators. Macromolecules 33, 4048-4052.

Kantchev, A.B., Parquette, J.R., 1999. Disaccharide synthesis on a soluble hyperbranched polymer. Tetrahedron Lett. 40, 8049-8053.

Kim, R.M., Manna, M., Hutchins, S.M. et al., 1996. Dendrimer-supported combinatorial chemistry. Proc. Natl. Acad. Sci. 93, 10012-10017.

Kleij, A.W., Ford, A., Jastrzebski, J.T.B.H., Van Koten, G. (in press). Dendritic polymer applications: catalysts. In: Fréchet, J.M.J., Tomalia, D. (Eds.), Dendritic Polymers.

Kreiter, R., Kleij, A.W., Klein Gebbink, R.J.M., Van Koten, G., 2001. Dendritic catalysts. In: Vögtle, F., Schalley, C. (Eds.), Dendrimers Topics in Current Chemistry, 217. Springer Verlag, Berlin, pp. 163-199.

Ley, S.V., Baxendale, I.R., Bream, R.N. et al., 2000. Multi-step organic synthesis using solid-supported reagents and scavengers: a new paradigm in chemical library generation. J. Chem. Soc. Perkin Trans. I, 3815-4195.

Lubineau, A., Malleron, A., Narvor, C.L., 2000. Chemo-enzymatic synthesis of oligosaccharides using a dendritic soluble support. Tetrahedron Lett. 41, 8887-8891.

Mahajan, A., Chhabra, S.R., Chan, W.C., 1999. Resin-bound dendrimers as high loading supports for solid-phase chemistry. Tetrahedron Lett. 40, 4909-4912.

Malenfant, P.R.L., Fréchet, J.M.J., 2000. Dendrimers as solubilizing groups for conducting polymers: preparation and characterization of polythiophene functionalized exclusively with aliphatic ether convergent dendrons. Macromolecules 33, 3634-3640.

Malenfant, P.R.L., Jayaraman, M., Fréchet, J.M.J., 1999. Dendrimer-supported oligothiophene synthesis: aliphatic ether dendrimers in the preparation of oligothiophenes with minimal substitution. Chem. Mater. 11, 3420-3422.

Marsh, A., Carlisle, S.J., Smith, S.C., 2001. High-loading scavenger resins for combinatorial chemistry. Tetrahedron Lett. 42, 493-496.

Merrifield, R.B., 1963. Solid-phase peptide synthesis. I. The synthesis of a tetrapeptide. J. Am. Chem. Soc. 85, 2149-2154.

Merrifield, R.B., 1969. Solid-phase peptide synthesis. Adv. Enzymol. 32, 221-296.

Newkome, G.R., Moorefield, C.N., Vögtle, F., 1996. Dendritic Molecules: Concept, Synthesis and Perspectives. VCH Verlag, Weinheim.

Oosterom, G.E., Reek, J.N.H., Kamer, P.C.J., Van Leeuwen, P.W.N.M., 2001. Transition metal catalysis using functionalized dendrimers. Angew. Chem. 40, 1828-1849.

Pirrung, M.C., 1997. Spatially addressable combinatorial libraries. Chem. Rev. 97, 473-488.

Reed, N.R., Janda, K.D., 2000. Stealth star polymers: a new high-loading scaffold for liquid-phase organic synthesis. Org. Lett. 2, 1311-1313.

Swali, V., Wells, N.J., Langley, J., Bradley, M., 1997. Solidphase dendrimer synthesis and the generation of superhigh-loading resin beads for combinatorial chemistry. J. Org. Chem. 62, 4902-4903.

Tam, J.P., 1988. Synthetic peptide vaccine design: synthesis and properties of a high-density multiple antigenic peptide system. Proc. Natl. Acad. Sci. USA 85, 5409-5413.

Thompson, L.A., Ellman, J.A., 1996. Synthesis and applications of small molecule libraries. Chem. Rev. 96, 555-600.

Tomalia, D.A., 1993. Starburst/cascade dendrimers: fundamental building blocks for a new nanoscopic chemistry set. Aldrichim. Acta 26, 91-101.

Toy, H.P., Janda, K.D., 2000. Soluble polymer-supported organic synthesis. Acc. Chem. Res. 33, 546-554.

Vögtle, F. (Ed.), 1998. Dendrimers. Topics in Current Chemistry, vol. 197. Springer Verlag, Berlin.

Vögtle, F. (Ed.), 2000. Dendrimers II: Architecture, Nanostructure and Supramolecular Chemistry. Topics in Current Chemistry, vol. 210. Springer Verlag, Berlin.

Wells, N.J., Davies, M., Bradley, M., 1998. Cleavage and analysis of material from single resin beads. J. Org. Chem. 63, 6430-6431.

Wells, N.J., Basso, A., Bradley, M., 1999. Solid-phase dendrimer synthesis. Biopolymers (Pept. Sci.) 47, 381-396.

Wentworth, P., Janda, K.D., 1999. Liquid-phase chemistry: recent advances in soluble polymer-supported catalysts, reagents and synthesis. Chem. Commun. 1917-1924. 